\title{
Article
}

\section{Adaptive Coding in Control of Two Satellites Relative Motion Over the Packet Erasure Communication Channel}

\author{
Boris Andrievsky ${ }^{1,2,4+,+*} \mathbb{C}$, Alexander L. Fradkov $1,2,3,5 \dagger, \pm \mathbb{C}$ and Elena V.Kudryashova ${ }^{3}$ \\ 1 Institute of Problems in Mechanical Engineering, Russian Academy of Sciences, Saint Petersburg, Russia; \\ b.andrievsky@spbu.ru \\ 2 Saint Petersburg University, Saint Petersburg, Russia; fradkov@mail.ru \\ 3 ITMO University, Saint Petersburg, Russia; e.kudryashova@ spbu.ru \\ 4 Baltic State Technical University, Saint Petersburg, Russia \\ 5 School of Automation, Hangzhou Dianzi University, Hangzhou, China \\ $\dagger$ Current address: Institute of Problems in Mechanical Engineering, Russian Academy of Sciences, 61 Bolshoy pr. V.O., \\ 199178, Saint Petersburg, Russia
}

\begin{abstract}
The paper is focused on the navigation data exchange between two satellites moved in a swarm. The feedback control law is designed ensuring regulation of the relative satellites motion. The adaptive binary coding/decoding procedure for data transmission over the limited capacity communication channel is proposed and studied for the cases of ideal and erasure channel. Dependence of the regulation time on the data transmission rate is numerically found. The results obtained provides dependence of the required load of the communication channel on the desired quality of the stabilization process. It is demonstrated that for significantly high data transmission rate erasure of data in the channel with probability up to 0.3 does not make an effect on the regulation time.
\end{abstract}

Keywords: data transmission; erasure communication channel; adaptive coding; satellite formation

\section{Introduction}

In the recent years, there has been a growing interest in using the differential force (i.e., the difference between the aerodynamic drag forces applied to the satellites) to eliminate the relative drift between the satellites in a swarm moving in a group (without the mandatory requirement to maintain relative position, cf. [1-3]). Various control algorithms using differential aerodynamic drag have been proposed in the numerous publications, see [4-16]. One of the fundamental publications is the work by Leonard [17] where, based on the assumption of the possibility of changing the effective cross-section of satellites, a method of switching control has been developed. The differential force is created by changing the attack angles $\alpha$ of the plates, located on the satellites, due to the rotation of the satellites with respect to the incident airflow.

For describing the dynamics of the relative motion of two satellites, moving in near-circular orbits at the Earth's central gravitational field, equations in the Local Vertical Local Horizontal (LVLH) reference system in relative coordinates according to the Hill-Clohessy-Witshire (HCW) model, see [18-23] were used.

In the present paper, the LVLH reference system is employed, where $\mathrm{OZ}$ axis is directed from the center of the Earth, OY axis is directed normal to the orbital plane, OX axis complements the triple to the right coordinate frame, see Fig. 1.

Taking into account that motion along the normal to the orbital plane (along OY axis) is isolated, and proceeding from the universal gravitation, law and Kepler's third law under the assumption that for distance $\rho$ of the satellite to the center of the Earth, inequalities $\rho x, z$ are hold, one obtains the following HCW equations of satellite motion in the LVLH local coordinate system:

$$
\begin{aligned}
& \ddot{x}=-2 \omega \dot{z}+F_{x}, \\
& \ddot{z}=2 \omega \dot{x}+3 \omega z+F_{z},
\end{aligned}
$$




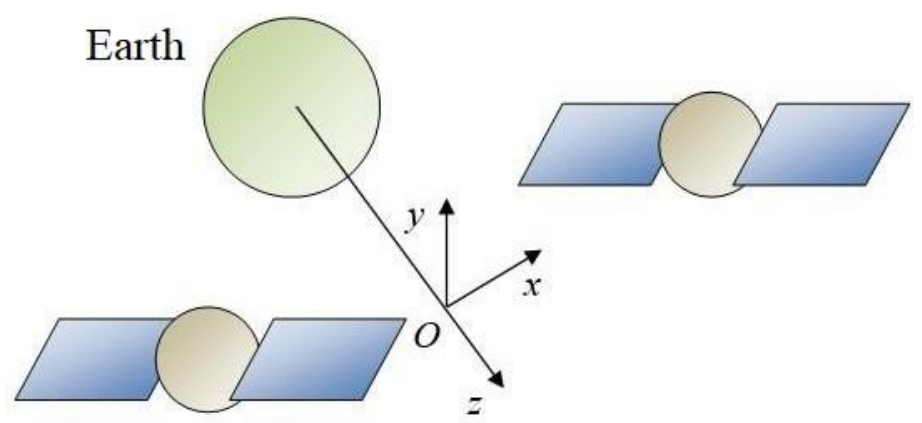

Figure 1. Coordinate system associated with reference point $\mathrm{O}$, moving in a circular orbit.

where $F_{x}, F_{z}$ are the components of the vector of non-gravitational forces applied to the satellite, expressed in acceleration units. These forces include the aerodynamic drag force $f_{x}$ along OX axis, which is controlled by the satellite turning through attack angle $\alpha$. Based on physical considerations it is valid thet $f_{x}=f_{x}(\alpha) \leq 0$, $f_{x}(0) \leq\left|f_{x}\right| \leq f_{x}(\pi / 2)$. Therefore it is natural considering angle $\alpha$ within $0 \leq \alpha \leq \pi / 2$. In (2), $\omega$ denotes the averaged angular velocity of the spacecraft in orbit and satisfyes the expression $\omega=\overline{\mu / a^{3}}$ where $\mu=G M$, $G$ is the gravitational constant, $M$ is the mass of the central body (for the Earth, $\mu=398603 \times 10^{9} \mathrm{~m}^{3} \times \mathrm{s}^{-2}$ ), $a$ is the semi-major axis of the satellite orbit. HCW equations (1), (2) are derived under the fassumptions: $\frac{\sqrt{x^{2}+z^{2}}}{x^{2}}$ is small compared to $\rho$; the aerodynamic force is small; the eccentricity of the orbit is small, the orbit is very close to the circular one; the orbital rate $\omega$ is approximately constant. For the resulting system (1), (2) in [17], a non-degenerate coordinate transformation is performed to the real Jordan form [24], as a result of which the model (1), (2) is represented in the form of a double integrator and a harmonic oscillator, connected in parallel. For the double integrator, a speed-optimal control is created in the form of a relay feedback with a quadratic switching function. A similar, but technically more complex approach is used for the harmonic oscillator. Since the control is scalar, it is not possible to apply both control laws simultaneously and independently to both the double integrator and the oscillator. To resolve this contradiction, Leonard [17] has developed and studied for different flight scenarios an algorithm for switching from one control law to another, based on the specifics of the rendezvous problem requirements.or different flight scenarios an algorithm for switching from one control law to another, based on the specifics of the rendezvous problem requirements.

Pérez and Bevilacqua [5] developed a robust approach based on the Lyapunov method to control the relative motion of two satellites using differential resistance. Based on the results of the works by Schweighart and Sedwick [21,25], see also [26, Chap. 2], a model of the dynamics of relative motion that takes into account the influence of $J_{2}$ perturbation on the spacecraft motion. ${ }^{1}$

$$
\begin{aligned}
& \ddot{x}+2 n c \dot{z}=F_{x}, \\
& \ddot{y}+q^{2} y=2 l q \cos (q t+\phi)+F_{y}, \\
& \ddot{z}-2 n c \dot{x}-\left(5 c^{2}-2\right) n^{2} z=F_{z},
\end{aligned}
$$

where

$$
n=\frac{\omega}{c}, \quad c=\overline{1+\frac{3 J_{2} R}{8 r_{T}^{2}} \cdot 1+3 \cos \left(2 i_{t}\right)^{\Sigma},}
$$

$i_{t}, r_{t}$ are inclinations and radii of the reference orbit of the target satellite, $i_{T}, r_{T}$ denote their initial values; $q$ is the linearized splitting argument in the transverse work plane, see [21]; $J_{2}$ is the coefficient of the second zonal harmonic of the Earth's gravitational potential, $J_{2}=1082.636023 \times 10^{-6}$; angle $\phi$ satisfies the equation $l \sin \phi+q y(0) \cot \phi=y^{\prime}(0)$ [23]. Pérez and Bevilacqua [5] proposed a control strategy in which a spacecrafts

1 The second zonal harmonic $\mathbf{J}_{2}$ characterizes the polar compression of the Earth [26]. 
formation tracks a given trajectory. The control sequence is constructed in such a way as to ensure the negativity of the time derivative of the Lyapunov function for the tracking error, and the spacecraft control system provides tracking of the reference model, which imposes restrictions on possible relative accelerations. Therefore, the desired trajectory for the approach maneuver can be obtained for a linear model using the linear control theory methods. The rendevous maneuver is divided into two successive phases [27,28]. On the first one, the spacecraft is launched into a stable relative orbit; on the second phase, the oscillation of the relative orbit stops and the conditions of approach are achieved, namely zero relative position and speed. For this purpose, the transformation is made of the state vector from LVLH coordinates to a new set of coordinates, in which the dynamics of the system is divided into a double integrator and a harmonic oscillator.

Kim et al. [29] consider a satellite constellation consisting of a leader and surrounding slave satellites. The orbit of the leader is considered as a reference one, and the relative orbits of the followers are considered to be the (Projected Circular Orbit, PCO). PCO is the relative orbit between the master and slave satellites, which is circular when it is projected onto the local horizontal plane [30]. PCO predicted distances [31] between master and slave satellites are is constant. The HCW equation describes the relative motion between the master and slave satellites in the local reference frame LVLH, with the origin on the master satellite.

In the recent publication by Traub et al. [15] it is noted that employment of differential aerodynamic forces is a promising option for satellite mission control without consuming propellant for a satellite flight. It is noted that adsorption of atomic oxygen on the surface of a satellite in very low Earth orbit causes diffuse reflection and high levels of energy accommodation, resulting in low lift coefficients, but surface materials that promote specular or quasi-specular reflections can significantly increase the magnitude of the available differential lifting forces. In [15] the robust control approach by Pérez and Bevilacqua [5], Omar and Bevilacqua [32] is employed for satellite mission control.

Monakhova and Ivanov [23] considered the problem of constructing a swarm of nanosatellites immediately after their separation from the launcher. To solve it, the decentralized control with using the force of aerodynamic drag in order to eliminate the drift between satellites is used in [23]. It is worth mentioning that in [23] the satellite control algorithm is not presented in a closed-loop form, but in the form of the duration of a constant control action depending on the initial conditions for excluding the time-linear component of the relative deviation, ignoring oscillatory one. From an application viewpoint, the solution of the control problem in the closed-loop form (according to the current measurements) is preferable and is covered by the method used in [17,27,28], where the system model is transformed to the Jordan form.

In [23], communication restrictions are taken into account in the sense of the radio signal fading with increasing the inter-satellite distance. This leads to the space limitation within which the position of neighboring vehicles may be transmitted between them and, consequently, to narrowing the number of satellites which are available for the navigation data exchange. However, the communication restrictions from an information-theoretical viewpoint are not taken into account in [23]: according to this work, since the radio communication between satellites is established, any amount of information can be transmitted over the communication channel per unit of time. In other words, the communication channel capacity in [23] is assumed to be infinitely large. However, in reality the data transmission rate is limited and, moreover, from an energy consumption viewpoint it is highly desirable to minimize the amount of data transmitted over the communication channel. Among other things, this approach can also make it possible increasing the power of transmitted signals, which, with an equal power consumption, may expand the area of inter-satellite interaction.

The limitations of control under constraints imposed by the limitations of communication channel capacity have been deeply studied the control theoretic literature, see [33-37] and the references therein. A fundamental result establishing the smallest value for which the stabilization (estimation) problem for linear time invariant (LTI) systems is obtained by Nair and Evans [33] and presented in the form of the seminal Data Rate Theorem.

In the present paper, a system of two coupled satellites is considered. As in [23], the satellites are assumed to be launched at the starting time in accordance to the specified separation conditions. It is assumed that the satellites move in a low circular near-Earth orbit (LEO) and are controlled using the aerodynamic drag force, which is achieved by rotating the satellite relative to the incoming flow using a flywheel attitude control system. The main focus of the paper is in the navigation data exchange between the satellites to be used to keep the 
satellites motion in a swarm. To this end, the adaptive coding procedure is proposed and is studied for the cases of ideal and erasure communication channel. The regulation time is taken as the performance criterion, and its dependence on the data transmission rate is numerically studied.

The reminder of the paper is organized as follows. Dynamics of the relative motion of two satellites in a near-circular orbit are described in Sec. 2. Section 3 deals with designing the control law for regulation of the satellites relative motion. Some key results of control and estimation under information constraints are briefly recalled in Sec. 4. Results of the numerical evaluations are presented in Sec. 5. Concluding remarks in Sec. 6 finalize the paper.

\section{Model of the dynamics of the relative motion of two satellites in a near-circular orbit}

In this study it is assumed that the onboard control system of each satellite is supplied by the values of all the relative coordinates $x_{12}=x_{2}-x_{1}, z_{12}=z_{2}-z_{1}$, and also their time derivatives. Control signals for each satellite are generated so as to provide the difference $u=u_{2}-u_{1}$, generated in accordance with the chosen control law $u=U\left(x_{12}, z_{12}, \dot{x_{12}}, z_{12}\right)$. It is supposed that each satellite can transfer position information to another one received by onboard navigation equipment, for example, using GLONASS / GPS receivers, installed on each [38] satellite and share this information via the digital inter-satellite communication channel.

Satellites moving in a circular near-earth orbit are considered. The linearized model of their relative motion in the OXZ plane is (cf. [23] and (3)-(5)) is as follows

$$
\begin{aligned}
& \dot{X}=A X+B u, \\
& u=-\phi(y), \\
& y=C X
\end{aligned}
$$

where $X={ }^{\Sigma} x_{12} \quad \dot{x}_{12} \quad z_{12} \quad z_{12}^{\Sigma^{T}} \in \mathrm{R}^{4}$ is the system state vector, where $x_{12}$ denotes the difference in coordinates of the second and first satellites along the OX axis, $x_{12}=x_{2}-x_{1} ; z_{12}=z_{2}-z_{1}$; $u$ denotes the control action (difference of aerodynamic forces acting on satellites, in units of acceleration). It is limited in modulus by the value $u_{\max }$. The equations (8), (9) close the feedback loop. In them, $y$ is the output of the linear controller in the feedback, $\phi(\times)$ is a nonlinear function describing the limitation of the control action, which is assumed to be the saturation function, $\phi(y)=\operatorname{sat}_{u_{\max }}(y)$. Since for each satellite the aerodynamic drag force is negative (that is, it acts against the direction of motion along the longitudinal axis and lies in the interval $\left[-u_{\max }, 0\right]$ ), then in order to provide the desired differential control $-u_{\max } \leq u_{i j} \leq u_{\max }$, the actual steering (braking) action should only be applied to the first (along X-axis) satellite of the pair, see [23].

The matrices of the dynamics model (7)-(9) have the form

$$
\begin{gathered}
A=\begin{array}{ccccc}
\square & \multicolumn{1}{c}{\square} & \square \square \\
0 & 1 & 0 & 0 & 0 \\
\square & 0 & 0 & -2 \omega \square \\
0 & 0 & 0 & 1 & \square \\
0 & 2 \omega & 3 \omega^{2} & 0 & \square 1 \square \\
\Sigma & 0 \square \\
\Sigma & \multicolumn{5}{c}{k_{1}} & k_{2} & k_{3} & k_{4},
\end{array}
\end{gathered}
$$

The coefficients $k_{i}, i=1, \ldots, 4$ are chosen at the stage of the control law synthesis.

\section{Control Law Design}

Let us use the pole-placement technique for designing the control law $u=U(X)$ by the modal control approach assuming that all the state variables $X_{i}, i=1,2$ are measured onboard of the each satellite and transferred to another one for calculating the relative position/velocity vector $X$. The design procedure is made for LTI system model under the assumption that the restriction on the control signal are not "active", i.e. $u$ does not go 
beyond the boundaries of the linear region, that is $|u| \leq u_{\max }$. The equations of state of the closed-loop system (disregarding disturbances) then have the form

$$
\dot{X}=(A-B K) X
$$

where the matrices $A, B, K$ are of the form (10).

The design problem consists in choosing the coefficients of the controller $k_{i}$ so as to provide the required spectrum $\left\{\lambda_{A B C}\right\}$ of the matrix $(A-B K)$ of the closed-loop system (11). The fourth order Butterworth polynomial

$$
D(s)=s^{4}+2.6131 \Omega s^{3}+3.4142 \Omega^{2} s^{2}+2.6131 \Omega^{3} s+\Omega^{4},
$$

is used, where parameter $\Omega$ is the geometric mean root of the characteristic polynomial. This parameter determines the desired transient time of the closed-loop system.

The present research is focused on the data exchange between the satellites, therefore other control schemes may be also employed. For example, in the case of essential parametric uncertainty, the Implicit Reference Model (IRM) adaptive control method [39,40] may be used.

\section{Control and Estimation under Information Constraints}

\subsection{Problem Description}

Let us consider control and observation (estimation) systems containing a digital communication channel. The structures of such systems are summarized in Figs. 2, 3.

In the considered control systems (Fig. 2) plant outputs $y(t)$, measured by the sensor at discrete instants $t_{k}$ $(k=0,1, \ldots)$, are converted by the coder $C$ into the characters of the coding alphabet $\mathrm{S}$, that are transmitted over a digital communication channel to the decoder $D$, which transforms messages from the form in which they were transmitted over the communication channel, in a form adequate to subsequent calculations and transformations by the controller. An example is the situation when the decoder tries to restore as far as possible the sensor readings $y(t)$, on the basis of which the transmitted over the channel were formed messages. The controller generates control action $u(t)$ based on the restored data. Since that some information is lost due to the finiteness of the alphabet $S$ and time sampling, a transmission error $\delta(t)$ appears. Note, that the measurement errors, conversion, and signal distortion during transmission over a communication channel lead to additional errors. In addition, in many tasks the delay in the communication channel should also be taken into account. In some cases, control signal $u(t)$ is also transferred to actuators over the digital communication channel. This also leads to coding signal $u(t)$ into the form, which is perceived by the communication channel (the corresponding encoder can be considered as part of the controller), and decoding at the other end of the channel into a form acceptable by an actuator. Thus, in a closed-loop control systems, discrete in time and level nonlinear devices appear that affect the system behavior.

The problem of design the control systems with limited bandwidth communication channels between individual components is close to that of designing digital systems with level quantization in the sense that for them one can express the amount of data transmitted between individual elements of the system in units of the amount of information. But there are very significant differences between them, see [35-37,41,42]. Indeed, in systems with level quantization, the characteristic converter (quantization step) is usually fixed, whilest the only restriction on the encoder is the number of characters in the alphabet used. Encoding function may change over time, depending on all previous measurements. This additional degree of freedom fundamentally changes the being the problem under consideration, since the data processing device (encoder) can effectively select the quantities to be measured by purposeful change of the coding function. Moreover, for data transmission over the communication channel has a delay, the value which grows linearly with the number of codeword bits. Therefore, starting from the some value, an increase in the number bits of the encoder can lead to a decrease in the accuracy of the estimation due to obsolescence of the transmitted data.

In [43-45] the communication channel was considered of limited capacity otherwise ideal. The cases of packet erasure channel and 'blinking' channel are widely appear in various real-world systems, see, e.g. [46-55]. 


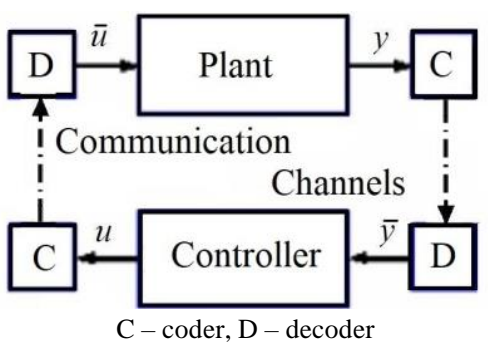

Figure 2. Structure of the control system through a digital communication channels.

In estimation systems (Fig. 3) it is required to find estimate $x^{\wedge}(t)$ of the plant state vector $x(t)$ based on the measurd plant output $y(t)$, wich is transmitted over a digital communication channel. Estimator (observer) transforms quantized data to estimate $x^{\wedge}(t)$. Separate interest in this regard has the situation when evaluated process $x(t)$ depends on some unmodeled signal $r(t)$ (which may be the control action, or the reference signal, e.g.). It is advisable to use this data for more accurate estimation (for example, based on Kalman filtering), however, there may be a need for transmission over the communication channel and measurements $r(t)$, which will lead to additional quantization errors and increasing the channel load.

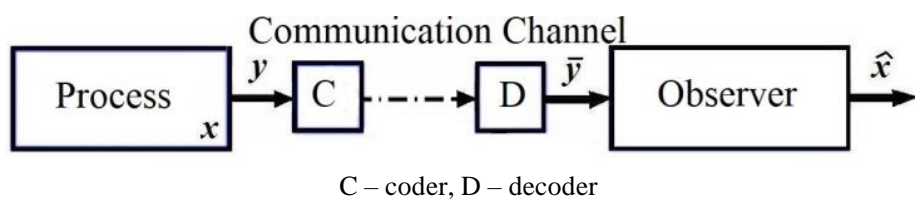

Figure 3. Structure of the observation system via digital communication channel.

Signal quantization introduces essentially non-linear properties into the system, characterized by the presence of the dead zone, discontinuities and saturation (associated with bit grid overflow). Additionally, the signal time sampling involves the hybrid (continuous-discrete) system decription. Rigorous analysis of the influence of time sampling and the level quantization is a complex nonlinear analysis problem, that usually has not an exact analytical solution. In the early studies, the level quantization in digital control systems was usually considered as a source of independent additive random noise affecting the system. This assumption makes it possible to significantly simplify the study of level quantized systems, especially for the LTI plants. However, if the quantization level is relatively high (for example, in the case of binary quantization), this can lead to the appearance of self-oscillations and even to divergent system behavior, see [56-59]. Therefore, to analyze the system, its nonlinear model is required. Besides, the possibility of the bit grid overflow may also eefect the quantizer, as a result of which the saturation is introduced to the control loop [41,60].

\subsection{Minimum Necessary Data Rate for Estimation and Control}

The limitation of the data transmission rate over the communication channel can be expressed in the informational terms. Assume that alphabet $S$ consists of $\mu$ elements. Then at each step $k=0,1, \ldots$ over the channel an amount of $\tilde{R}=\log _{2} \mu$ bit can be transmitted per each step. Let the data transmission be carried out at discrete instants $t_{k}=k T_{0}$, where $T_{0}$ is the sampling interval. Then the data transmission rate in bits per second is as $R=T_{0}^{-1} \log _{2} \mu$ bit/s. In this regard, one speaks of "information constraints" in control and estimation problems.

For the Authors' best knowledge, the problem of determining the minimum bandwidth of the communication channel, at which it is possible to provide the required estimation accuracy, is posed and partially solved by Nair and Evans [61]. The sufficient condition for the value obtained in Nair and Evans [61] was developed in subsequent works in the form of the Data Rate Theorem which is a fundamental result establishing the smallest value for which the stabilization (estimation) problem for linear systems is solvable in principle. Nair and Evans [33] studied the exponential stabilizability of LTI plants in the sense of achieving an exponential moment stability. For a deterministic initial state case the result of [33] can be roughly presented in the following form [42]. 
Let the LTI discrete-time plant be described by the difference equation

$$
x[k+1]=A x[k]+B u[k], \quad y[k]=C x[k], k=0,1, \ldots
$$

where $x[k] \in \mathbf{R}^{n}, y[k] \in \mathbf{R}^{l}, u[k] \in \mathbf{R}^{m}$ are the state, output and control vectors, respectively; $A, B, C$ are the matrices of the corresponding dimensions; $k \in Z_{+}$denotes the step number (the discrete time). It is assumed that pair $(A, B)$ is reachable, $(C, A)$ is observable. Let the sensor be connected to the controller over a digital communication channel (see Figs. 2, 3), and no more than $\tilde{R}$ bits of data can be transmitted at each step $k$. Then the necessary and sufficient conditions for $\rho$-exponential (with the prespecified stability bound $\rho>0$ ) stabilization are given by inequality [33]:

$$
\tilde{R}>\sum_{\eta_{j} \geq \rho} \log _{2} \cdot \underline{\eta}_{i} \cdot \quad \text { (bit per step) }
$$

where $\eta_{j}$ are the eigenvalues of matrix $A, j=1, \ldots, n$. The right-hand side of (14), denoted as $\tilde{R}_{N E}=$ $\sum_{\eta_{j} \geq \rho} \log _{2} \cdot \underline{\eta}_{i}$. (the Nair-Evans-, or $N E$-number), gives a tight admissible bound when the $\rho$-exponential stabilization can be achieved. For real-time systems with constant sampling interval $T_{0}$, NE-number $R_{N E}$ in bits per second has a form

$$
R_{N E}=\frac{1}{T_{0}} \tilde{R}_{N E}=\frac{1}{T_{0}} \sum \log _{\eta_{j} \geq \rho} \cdot \underline{\eta}_{j} \cdot \rho . \quad \text { (bit per second). }
$$

Extensions of this result for stabilizing nonlinear systems in the vicinity of the origin and observing nonlinear systems through finite capacity communication channels, including large networks, were obtained in the series of the subsequent papers, see [62-66] to mention a few.

It is worth mentioning that on practice, the data transmission rate can not be taken as small as the NE-number (15) gives; due to the several reasons the data bitrate is usually much greater than $R_{N E}$, but the NE-number can serve as a measure showing the maximum available possibilities of estimation and control over the existing communication channel. Also a promising approach is to employ event-triggered control instead of control with constant sampling time, see e.g. [67-69].

\subsection{Coding/Decoding Schemes}

Under the assumption that the sampling time $T_{0}$ may be chosen arbitrarily, optimality of the binary coding in the sense of the required transmission rate (in bit-per-second) has been found by Fradkov et al. [45], cf. [70]. Therefore in the present study the binary quantizer is used as a core element for coding procedure.

\subsubsection{Static Binary Quantizer}

Let $\sigma[k]$ be a scalar information signal to be transmitted over the digital communication channel in discrete instants $t_{k}=k T_{0}$, where $k=0,1, \cdots \in Z_{+}$is a sequence of natural numbers, $T_{0}$ is the sampling interval. Let us introduce the following static quantizer

$$
q(\sigma, M)=M[k] s, \quad s=\operatorname{sign}(\sigma)
$$

where $\operatorname{sign}(\times)$ is the signum function: $\operatorname{sign}(\sigma)=1$, if $\sigma \geq 0, \operatorname{sign}(\sigma)=-1$, if $\sigma<0$. Parameter $M$ is referred to as a quantizer range. The output signal of the quantizer is represented as one-bit information symbol from the coding alphabet $S=\{-1,1\}$, and is transmitted to the decoder. Note that for the binary coder, the transmission rate is as $R=1 / T_{0}$ bit per second. It is assumed that the equi-memory condition is fulfilled, i.e. the coder and decoder make decisions based on the same information [71,72]. The binary output codeword $s \in S$ is transmitted to the decoder. 


\subsubsection{Zooming strategies}

In time-varying quantizers [35,45,73-76] range $M$ is updated with time. Using such a zooming strategy improves the steady-state accuracy of the transmission procedure and at the same time prevents the encoder saturation at the process beginning. The values of $M[k]$ may be precomputed (the time-based zooming) [45,77,78], or current quantized measurements may be used at each step for updating $M[k]$ (the event-based zooming). For an audio channel, Moreno-Alvarado et al. [79] developed the coding schemes with the capacity to simultaneously encrypt and compress audio signals, which makes possible increasing necessity for transmitting sensitive audio information over insecure communication channels.

The event-based zooming can be realized in the form of the adaptive zooming [80-83], where quantizer's range is adjusted automatically depending on the current variations of the transmitted signal.

For the binary quantizer the following adaptive zooming algorithm was proposed and experimentally studied in [82]:

$$
\begin{aligned}
& \lambda[k]=(s[k]+s[k-1]+s[k-3]) / 3, \quad s[-1]+s[-2]=0, \\
& M[k+1]=m_{c}+\quad \rho M[k], \quad \text { if }|\lambda[k]| \leq 0.5, \\
& \sigma^{-}[k]=M[k] / \rho, \text { else, }
\end{aligned}
$$

where $M[0]=M_{0}$ is an initial value of $M[k]$ (the design parameter); $\sigma^{-}[k]$ denotes the value of $\sigma[k]$ recovered at the decoder side from binary values $s[k]$. Unlike the case of time-based zooming, the initial value $M_{0}$ can be chosen arbitrarily, since $M[k]$ is automatically adjusted and can be increased or decreased during the zoom-in and zoom-out stages.

\subsubsection{Coders with Memory}

The coding/decoding procedure may employ the embedded observer, which adds a memory to the coder. The following drive process model is used:

$$
x \cdot(t)=A x(t)+B \phi(t), y(t)=C x(t), x(0)=x_{0}
$$

where $x(t) \in \mathrm{R}^{n}$ is the process state space vector; $y(t)$ is the scalar measured signal; $A \in \mathrm{R}^{n \times n}, B \in \mathrm{R}^{n \times 1}$ are known real matrices; $\phi(t)$ is the external input signal which is assumed to be the same both on the transmitter and receiver sides (so that the equi-memory condition be fulfilled).

The quantized observation error $\sigma^{-}[k]$ is defined as a deviation between measured signal $y(t)$ and its estimate $y^{\wedge}(t)$ quantized with given $M[k]$ as follows:

$$
\bar{\sigma}[k]=q \dot{y}\left(t_{k}\right)-y^{\wedge}\left(t_{k}\right), M[k]^{\Sigma}, t_{k}=k T .
$$

where the estimate $y^{\wedge}(t)$ is generated by the following observer

$$
\dot{\hat{x}}(t)=A \hat{x}(t)+B \dot{\phi}^{-} \hat{y}(t)^{\Sigma}+L \bar{\sigma}(t), \hat{y}(t)=C \hat{x}(t),
$$

where $x^{\wedge}(t) \in \mathrm{R}^{n}$ is the state estimation vector; $y^{\wedge}(t)$ is estimate of the drive process; $L$ is $(n \times 1)$-matrix (the column-vector) of the observer parameters; continuous-time observation error $\bar{\sigma}(t)$ is found as an extension of $\bar{\sigma}[k]$ over the sampling interval. In the case of the zero-order extrapolation, $\bar{\sigma}(t)$ has a form $\bar{\sigma}(t)=\bar{\sigma}[k]$ as $t_{k} \leq t<t_{k+1}$.

\subsubsection{Adaptive Coding for Transmission of Position Between Satellites in Formation}

Application of the adaptive coding for navigation data transmission between satellites in the formation is often based on the kinematic representation of the vehicle motion by the second-order model for each channel 
under the assumption that the satellite speed is constant, which leads to the following representation of the data source generator (cf. [84-86])

$$
\begin{aligned}
& y[k+1]=y[k]+T_{0} V[k], \\
& V[k+1]=V[k]+\xi[k],
\end{aligned}
$$

where $y[k], V[k]$ are the satellite position and speed with respect to the given direction, variable $\xi[k]$ denotes the unmodeled variations of the vehicle speed and is considered as an unknown disturbance.

To estimate the change of the coded signal, the following embedded observer is introduced to the encoder and decoder ([82,86]):

$$
\begin{aligned}
& y^{\wedge}[k+1]=y^{\wedge}[k]+T_{0} V^{\wedge}[k]+l_{1} \sigma[k], \\
& V^{\wedge}[k+1]=V^{\wedge}[k]+l_{2} \sigma[k],
\end{aligned}
$$

where $y^{\wedge}[k], \hat{V}[k]$ are the estimates of $y^{\wedge}[k], \hat{V}[k]$, respectively, $l_{1}, l_{2}$ are the observer design parameters.

\subsection{Erasure channel description}

By analogy with [53,86,87], let us assume that output measurement is encoded by an encoder and transmitted to a decoder through packet erasure channel with erasure probability $p$. Also suppose that there exists a feedback from decoder to the encoder for acknowledgment whether the packet was erased or not. Therefore the encoder knows what information has been delivered to the decoder (i.e. the aforementioned equi-memory condition is fulfilled). Let the acknowledgment signal at time $k$ which is sent by the decoder and received by the encoder be represented by $\zeta[k] \in\{0,1\}$ as follows:

$$
\zeta[k]=\begin{aligned}
& 0, \text { no erasure at instant } k \\
& 1, \text { otherwise. }
\end{aligned}
$$

Random variables $\zeta[k], k=0,1, \ldots$ are assumed to be independent and identically distributed with common distribution: $P_{r}(\zeta[k]=0)=1-p$ and $P_{r}(\zeta[k]=1)=p$.

\section{Numerical Study}

\subsection{Satellite Model Parameters}

For the numerical investigations two satellites are represented by $3 \mathrm{U}$-cubesates in the form of rectangular parallelepipeds with dimensions $10 \mathrm{~cm} \times 10 \mathrm{~cm} \times 30 \mathrm{~cm}$. The aerodynamic drag force acting to $i$-th satellite in the acceleration units is given by the relation $f \quad \rho V^{2} \quad \Delta S \sin \alpha \quad S m^{-1} \mathrm{~ms}^{-2}$ where $V$ denotes the $i=-{ }_{a} C_{a}\left(i-{ }_{0}\right)$

satellite running speed with respect to the Earth's atmosphere; $\rho=\rho(h)$ is the air density on the height $h$ of the satellite orbit; $\alpha_{i} \in[0, \pi / 2]$ stands for the angle of attack; $C_{\alpha}$ is the drag derivative coefficient with respect to attack angle; $\Delta S$ denotes the difference between the satellite cross-sectional areas for the cases of $\alpha=0$ and $\alpha=\pi / 2 \mathrm{rad} ; S_{0}$ is the cross-sectional area as $\alpha=0 ; m$ is the mass of the satellite. Therefore the differential drag may be found as $f \quad \rho V^{2} C \Delta S \sin \alpha \quad \sin \alpha$, which dives the following maximal control action

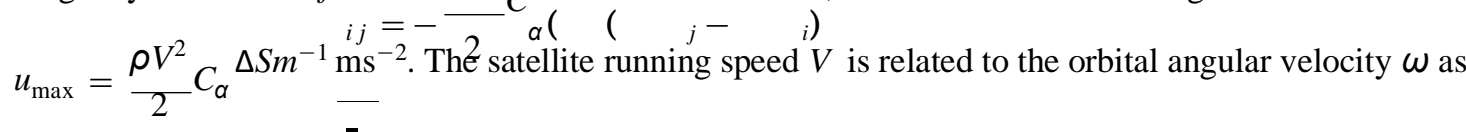
follows: $V=\omega r_{0}={ }_{r_{0}}^{-} \underline{\mu}$, where $\mu=M_{\text {earth }} G, G=6.6743 \times 10^{-11} \mathrm{~m}^{3} \mathrm{~kg}^{-1} \mathrm{~s}^{-2}, M_{\text {earth }}=5.972 \times 10^{24} \mathrm{~kg}$, so that $\mu=3.986 \times 10^{14} \mathrm{~m}^{3} \mathrm{~s}^{-2} ; r_{0}=R_{\text {earth }}+h, R_{\text {earth }}=6.371 \times 10^{6} \mathrm{~m}$.

The following values are taken for the numerical study: $\Delta S=0.02 \mathrm{~m}^{2}, C_{\alpha}=2, \rho=6.06 \times 10^{-11} \mathrm{~kg} / \mathrm{m}^{3}, h=$ $250 \times 10^{3} \mathrm{~m}, m=2 \mathrm{~kg}$. These parameters lead to $V=7.76 \times 10^{3} \mathrm{~m} / \mathrm{s}, \omega=0.001172 \mathrm{rad} / \mathrm{s} . u_{\max }=2.4 \times 10^{-5} \mathrm{~m} / \mathrm{s}^{2}$.

For controller design, parameter $\Omega$ in (12) is taken as $\Omega=10^{-3} \mathrm{rad} / \mathrm{s}$. The pole-placement technique [24]

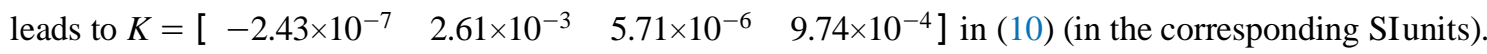




\subsection{Simulations for Ideal Communication Channel}

Consider the "ideal" case where the position information is transferred over the channel without sampling and level quantization. For the performance criterion let as take the instant $T^{*}$ when the relative trajectory on the $(X, Z)$ plane reaches the circle with given radius $Q$ and does not leave it in the future, i.e. $T^{*}=$

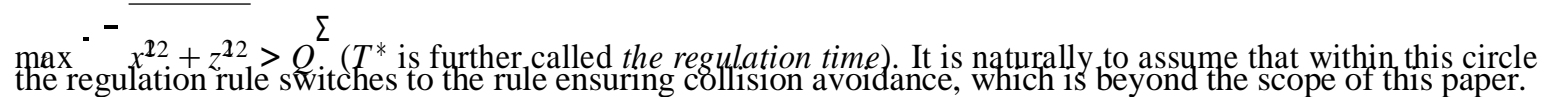

The simulation results for various initial conditions are depicted in Figs. 4-7. Figures 4, 5 are related to the case of $x_{12}(0)=20 \mathrm{~m}, \dot{x_{12}}(0)=0.05 \mathrm{~m} / \mathrm{s}$ and other initial conditions are zero. Time histories of relative positions $x_{12}(t), z_{12}(t)$ and control action $u(t)$ are plotted in Fig. 4. Symbol "+" marks regulation time $T^{*}$. For the given initial conditions, $T^{*}=3.27$ hours is obtained. Figures 6, 7 show similar plots for the case of $x_{12}(0)=200 \mathrm{~m}, \dot{x_{12}}(0)=0.025 \mathrm{~m} / \mathrm{s}, z_{12}(0)=-50 \mathrm{~m}, \dot{z}_{12}(0)=-0.025 \mathrm{~m} / \mathrm{s}$. In this case, $T^{*}=4.61$ hours.
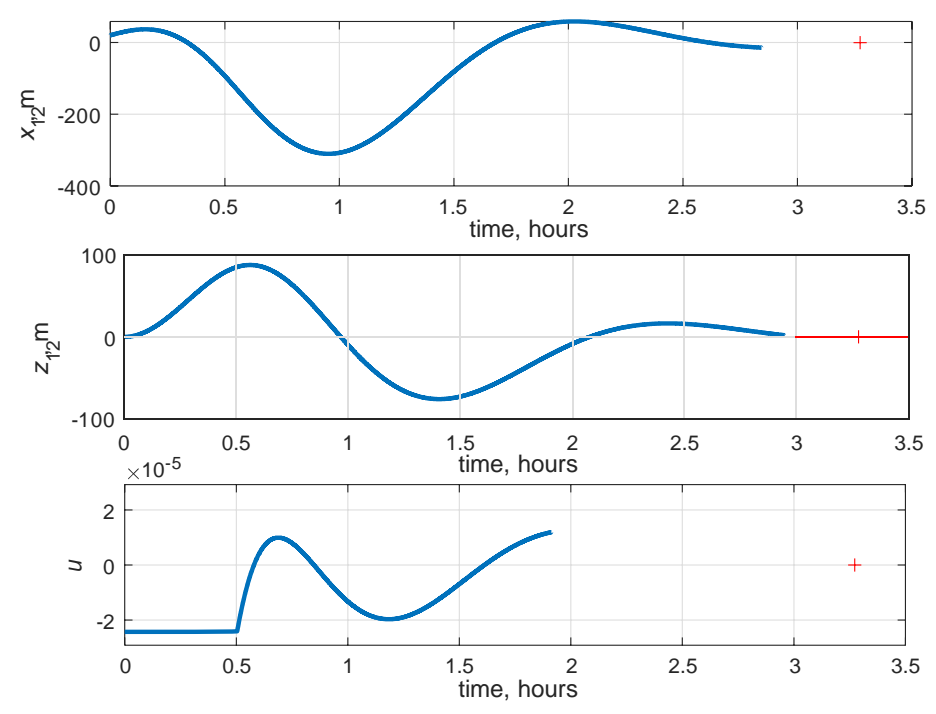

$x_{12}(0)=20 \mathrm{~m}, \dot{x}_{12}(0)=0.05 \mathrm{~m} / \mathrm{s} ; T^{*}=3.27$ hours

Figure 4. Time histories of $x_{12}(t)$ (upper plot), $z_{12}(t)$ (middle plot), $u(t)$ (lower). The ideal channel case.

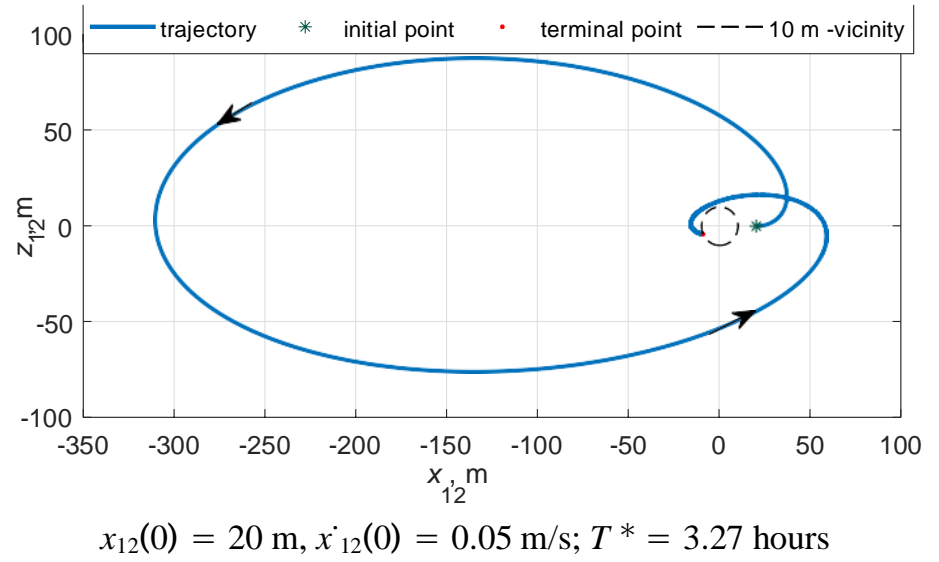

Figure 5. Satellites relative trajectories on (XZ) plane. The ideal channel case.

The simulation results show that, despite of the control signal saturation at the beginning of the process, the targeting manifold is attained for both sets of the initial conditions with regulation time $T^{*}=3.27$ hours (Figs. 4, 5) and $T^{*}=4.61$ hours (Figs. 6, 7), respectively. Moreover, based on the harmonic balance method arguments $[58,59]$ the conclusion can be made that the closed-loop system is globally asymptotically stable. 

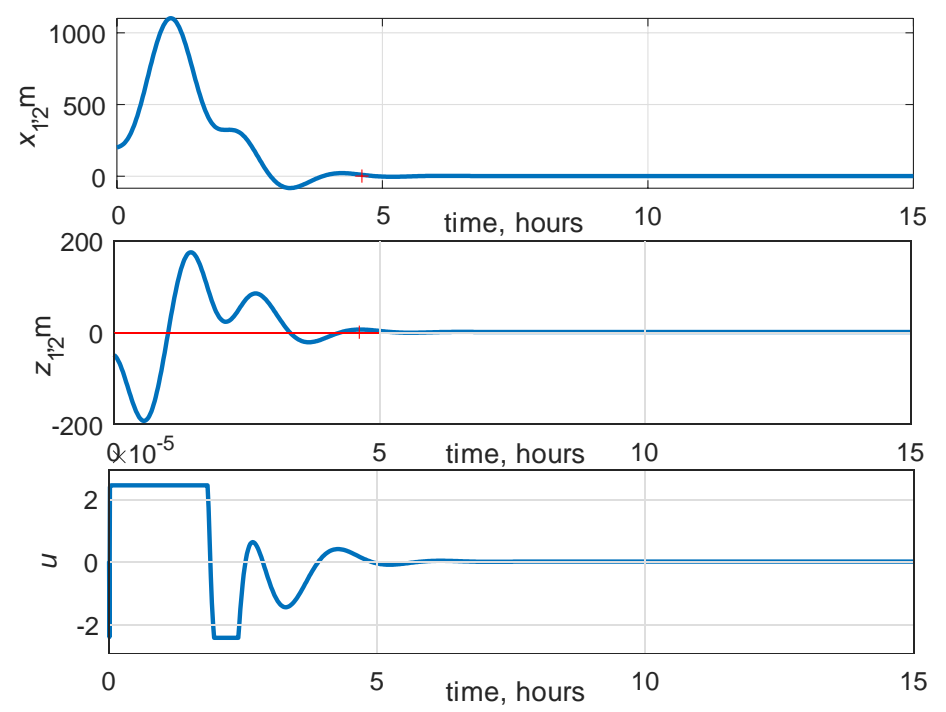

$x_{12}(0)=200 \mathrm{~m}, \dot{x}_{12}(0)=0.025 \mathrm{~m} / \mathrm{s}, z_{12}(0)=-50 \mathrm{~m}, z_{12}(0)=-0.025 \mathrm{~m} / \mathrm{s} ; T^{*}=4.61$ hours

Figure 6. Time histories of $x_{12}(t)$ (upper plot), $z_{12}(t)$ (middle plot), $u(t)$ (lower plot). The ideal channel case.

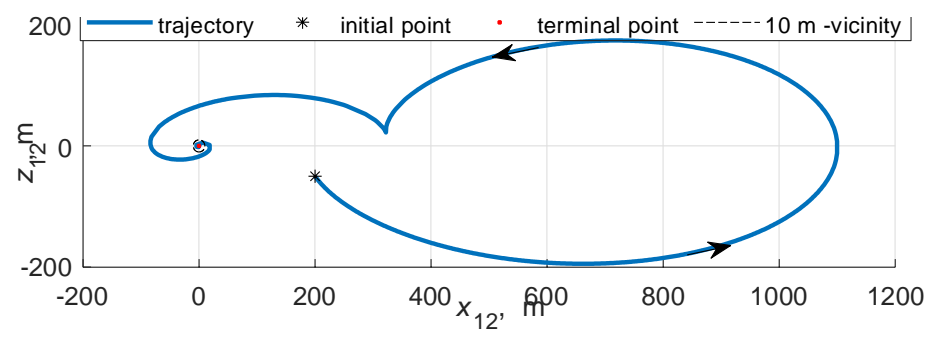

$x_{12}(0)=200 \mathrm{~m}, \dot{x_{12}}(0)=0.025 \mathrm{~m} / \mathrm{s}, z_{12}(0)=-50 \mathrm{~m}, z_{12}(0)=-0.025 \mathrm{~m} / \mathrm{s} ; T^{*}=4.61$ hours

Figure 7. Satellites relative trajectories on (XZ) plane. The ideal channel case.

\subsection{Satellite Position Transmission Over the Digital Communication Channel with Finite Capacity}

Consider now the case when the satellites exchange each other their LVLH coordinates over the binary communication channel, described in Sec. 4.2. It is assumed that each satellite transmits its coordinates $x_{i}$, $z_{i}, i=1,2$ to the associated one, where the estimates $x_{i}^{\wedge}[k], z_{i}[k]$ and $x_{i}[k], z_{i}[k]$ are obtained. The following quantization and data transmission algorithms are used: binary quantization (16), adaptive zooming (17), embedded state estimation (22). For the numerical study, the coding-decoding procedure parameters are picked up as follows: $M_{0}=1, m_{c}=0, \rho=e^{-0.01 T_{0}}$, gains $l_{1}, l_{2}$ of observer (22) are found by the pole-placement technique for the discrete-time system $\left.y^{\hat{[}} k+1\right]=y^{\hat{n}}[k]+T_{0} \hat{V}[k]+l_{1} \sigma_{i}[k], \hat{V}[k+1]=\hat{V}[k]+l_{2} \sigma_{i}[k]$, where $y[k]$ and $V[k]$ are related to estimates $x_{i}^{\wedge}[k], z_{i}^{\wedge}[k]$ and $x^{\wedge}{ }_{i}[k], z_{i} \cdot[k]$ depending on the transmitted coordinate $(x$ or $z)$ and the satellite number $i=1,2$. Initial conditions $y[0], V[0]$ are set to zero for all four channels. Therefore, two data transmission channels are supposed to be used on each satellite, so the data transmission rate for each binary communication channel $R=1 / T_{0}$ should be doubled to find the overall channel load.

The simulation results for $T_{0}=0.667 \mathrm{~s}\left(R=1.5 \mathrm{bit} / \mathrm{s}\right.$ for each transmitted from $i$-th satellite coordinate $x_{i}$ and $\left.z_{i}\right)$ and initial conditions $x_{12}(0)=200 \mathrm{~m}, \dot{x_{12}}(0)=0.025 \mathrm{~m} / \mathrm{s}, z_{12}(0)=-50 \mathrm{~m}, \dot{z_{12}}(0)=-0.025 \mathrm{~m} / \mathrm{s}$ are plotted in Figs. 8, 9, where time histories of $x_{12}(t), z_{12}(t), u(t)$ and the satellites relative trajectories on (XZ) plane are depicted. Regulation time $T^{*}=4.84$ hours is found by the simulation, which is a bit greater than the regulation time for non-restricted communication channel ( $T^{*}=4.61$ hours), compare with the plots on Figs. 6, 7. The time histories and trajectories for the ideal and the limited capacity communication channels differ due to the coder transient at the beginning of the coding procedure, which leads to oscillating control action during the initial data transmission time interval. Some measures (filtering of the control signal at the beginning of the process, or delayed start of stabilization) can be used for practical purposes. 

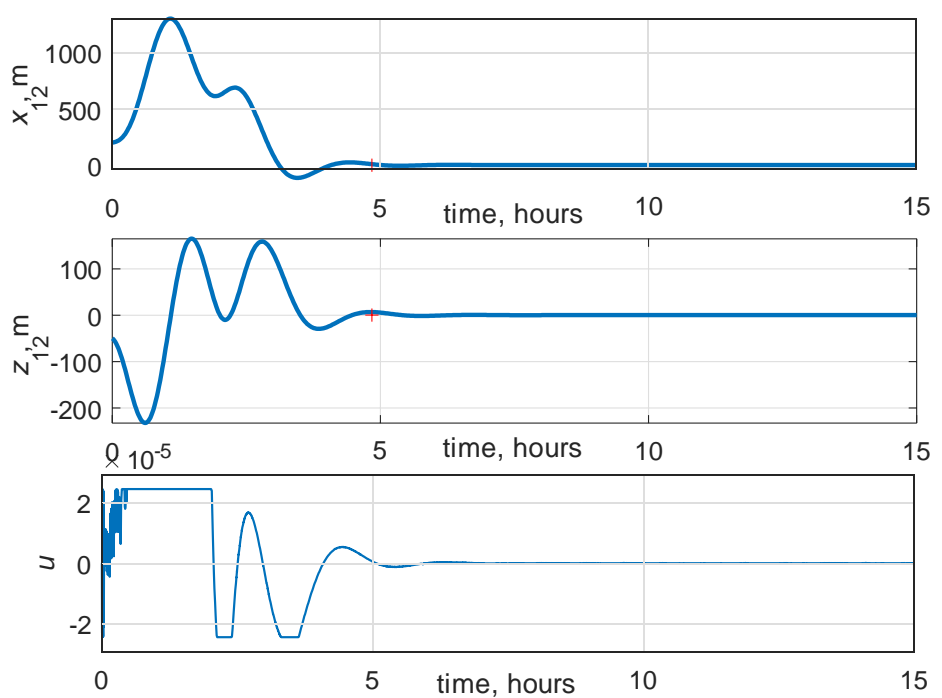

$x_{12}(0)=200 \mathrm{~m}, \dot{x}_{12}(0)=0.025 \mathrm{~m} / \mathrm{s}, z_{12}(0)=-50 \mathrm{~m}, \dot{z}_{12}(0)=-0.025 \mathrm{~m} / \mathrm{s} ; T^{*}=4.84$ hours.

Figure 8. Time histories of $x_{12}(t)$ (upper plot), $z_{12}(t)$ (middle plot), $u(t)$ (lower plot). Case of quantization, $R=1.5 \mathrm{bit} / \mathrm{s}, p=0$.

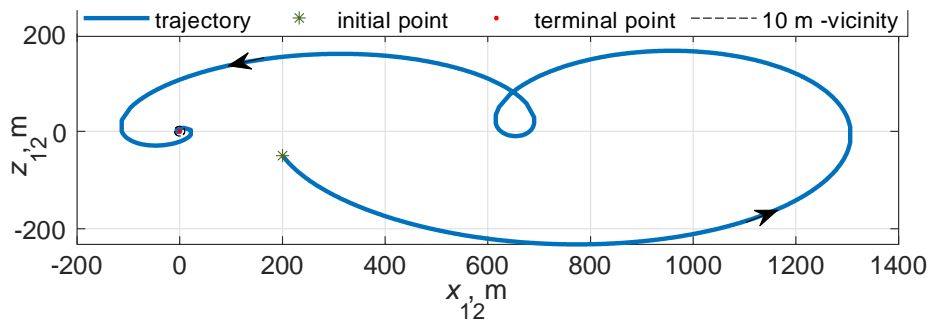

$x_{12}(0)=200 \mathrm{~m}, \dot{x_{12}}(0)=0.025 \mathrm{~m} / \mathrm{s}, z_{12}(0)=-50 \mathrm{~m}, z_{12}(0)=-0.025 \mathrm{~m} / \mathrm{s} ; T^{*}=4.84$ hours.

Figure 9. Satellites relative trajectories on (XZ) plane. Case of quantization, $R=1.5 \mathrm{bit} / \mathrm{s}, p=0$.

\subsection{Case of Erasure Communication Channel}

Let us consider now how the erasure of data in the communication channel affects the process of stabilizing the relative position of the satellites. The corresponding simulation results for $T_{0}=0.667 \mathrm{~s}$, initial conditions $x_{12}(0)=200 \mathrm{~m}, \dot{x_{12}}(0)=0.025 \mathrm{~m} / \mathrm{s}, z_{12}(0)=-50 \mathrm{~m}, z_{12}(0)=-0.025 \mathrm{~m} / \mathrm{s}$ and probability $p=0.2$ of erasing data in the communication channel are plotted in Figs. 10, 11. Regulation time $T^{*}=7.07$ hours is found by the simulation. The time histories and trajectories for the ideal and the limited capacity erasure communication channels significantly differ from the case of the ideal channel, and from an application viewpoint, the process quality for these conditions is a boundary one.

Illustrations of the adaptive coding procedure are given in Figs. 12, 13. Adaptively tuning quantizer range $M[k]$ in accordance with algorithm (17) is depicted in Fig. 12. The plot shows how the range is automatically increased at the initial stage of the process and decays then, which leads to reducing the data transmission error. Figure 13 demonstrates the influence of data erasure on the codewords, transmitted over the channel. Signal $s[k]$ from the coder is received in the form of $s_{\zeta}[k]$ on the decoder side. The difference between these signals causes the additional data transmission errors. A summary graph of the dependence of regulation time $T^{*}$ on the data transmission rate $R$ (for each one channel) at various erasure probabilities $p \in\{0,0.1,0.2,0.3\}$ is shown in Fig. 14 . The plots make an impression about the required load of the communication channel and the quality of the stabilization process with its use. It is demonstrated, that, as usual, the data transmission error depends inversely proportional on the communication rate. It is also seen from the plot that for significantly high data transmission rate erasure of data in the channel with probability up to 0.3 does not make an effect on the regulation time, and the regulation time is defined by the system dynamics for the case of the unbounded communication channel capacity. 

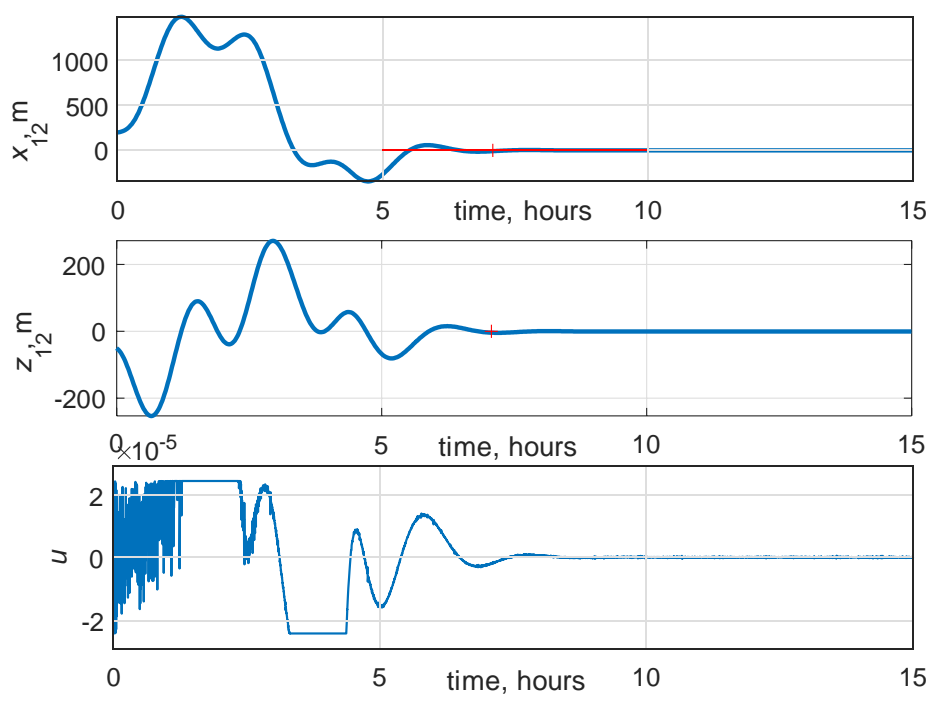

$x_{12}(0)=200 \mathrm{~m}, \dot{x}_{12}(0)=0.025 \mathrm{~m} / \mathrm{s}, z_{12}(0)=-50 \mathrm{~m}, z_{12}(0)=-0.025 \mathrm{~m} / \mathrm{s} ; T^{*}=7.07$ hours.

Figure 10. Time histories of $x_{12}(t)$ (upper plot), $z_{12}(t)$ (middle plot), $u(t)$ (lower plot). Case of quantization and erasure, $R=1.5 \mathrm{bit} / \mathrm{s}, p=0.2$.

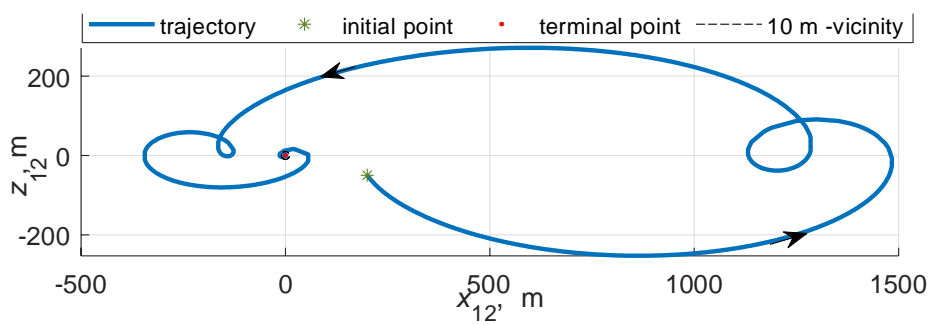

$x_{12}(0)=200 \mathrm{~m}, x_{12}(0)=0.025 \mathrm{~m} / \mathrm{s}, z_{12}(0)=-50 \mathrm{~m}, \dot{z}_{12}(0)=-0.025 \mathrm{~m} / \mathrm{s} ; T^{*}=7.07$ hours.

Figure 11. Satellites relative trajectories on (XZ) plane. Case of quantization and erasure, $R=1.5 \mathrm{bit} / \mathrm{s}, p=0.2$.

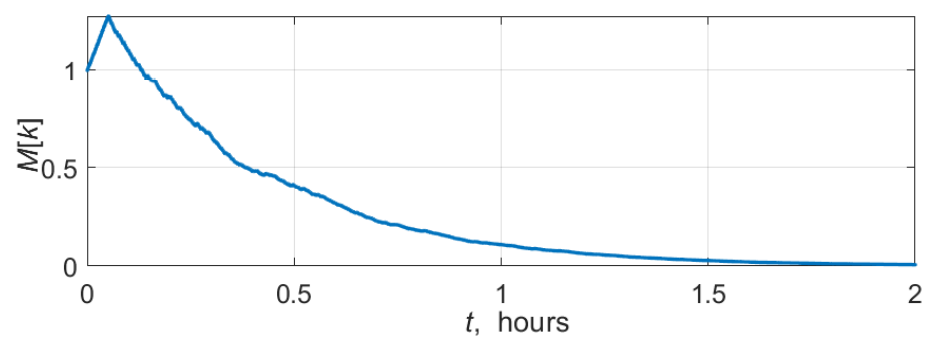

$x_{12}(0)=200 \mathrm{~m}, \dot{x}_{12}(0)=0.025 \mathrm{~m} / \mathrm{s}, z_{12}(0)=-50 \mathrm{~m}, \dot{z}_{12}(0)=-0.025 \mathrm{~m} / \mathrm{s} ; T^{*}=7.07$ hours.

Figure 12. Quantizer range $M[k]$ time history for the case of quantization and erasure, $R=1.5 \mathrm{bit} / \mathrm{s}, p=0.2$.

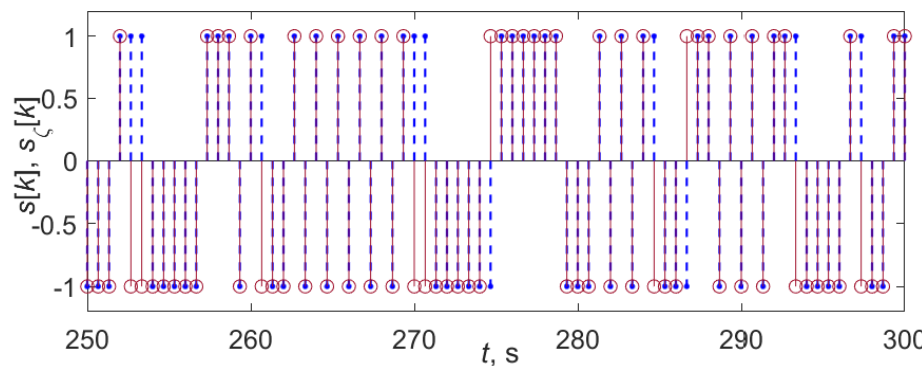

$x_{12}(0)=200 \mathrm{~m}, \dot{x}_{12}(0)=0.025 \mathrm{~m} / \mathrm{s}, z_{12}(0)=-50 \mathrm{~m}, z_{12}(0)=-0.025 \mathrm{~m} / \mathrm{s} ; T^{*}=7.07$ hours.

Figure 13. Time histories of $s[k]$ on the coder side (marked by the red circles) and $s_{\zeta}[k]$ (marked by the blue dots) on the decoder side. Case of quantization and erasure, $R=1.5 \mathrm{bit} / \mathrm{s}, p=0.2$. 


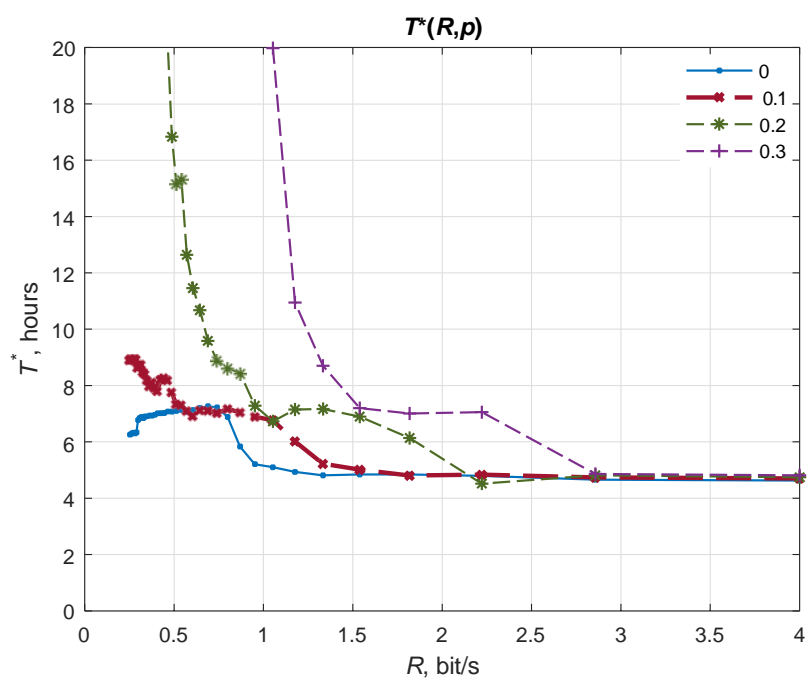

Figure 14. Regulation time $T^{*}$ vs transmission rate $R$ for various erasure probability $p$ values.

\section{Conclusions}

In the paper the feedback control law is designed ensuring regulation of the relative satellites motion in a swarm. The main purpose of the paper is investigating the navigation data exchange between the satellites . The adaptive binary coding/decoding procedure for data transmission over the limited capacity communication channel is proposed and studied for the cases of ideal and erasure channel. Dependence of the regulation time on the data transmission rate is numerically found. The results obtained provides dependence of the required load of the communication channel on the given quality of the stabilization process. Particularly, it is demonstrated, that the data transmission error depends inversely proportional on the communication rate.

Author Contributions: Conceptualization, B.A. and A.F; Data curation, B.A.; Formal analysis, E.K.; Funding acquisition, A.F.; Investigation, B.A.; Methodology, A.F.; Project administration, A.F.; Software, B.A. and E.K.; Supervision, A.F; Writing - original draft, B.A. Writing - review \& editing, B.A. and A.F. All authors have read and agreed to the published version of the manuscript.

Funding: This work was supported in part by the Government of Russian Federation (Grant 08-08) and by the Ministry of Science and Higher Education of Russian Federation (Grant FZWF-2020-0015).

Conflicts of Interest: The authors declare no conflict of interest.

\section{Abbreviations}

The following abbreviations are used in this manuscript:

LTI Linear Time Invariant

\section{References}

1. Chang, I.; Chung, S.J.; Blackmore, L. Cooperative control with adaptive graph Laplacians for spacecraft formation flying. Proc. IEEE Conf. Decision and Control (CDC 2010), 2010, pp. 4926-4933. doi:10.1109/CDC.2010.5717516.

2. Morgan, D.; Chung, S.J.; Blackmore, L.; Acikmese, B.; Bayard, D.; Hadaegh, F. Swarm-keeping strategies for spacecraft under $\mathrm{J} 2$ and atmospheric drag perturbations. Journal of Guidance, Control, and Dynamics 2012, 35, 1492-1506. doi:10.2514/1.55705.

3. Monakhova, U.; Ivanov, D.; Roldugin, D. Magnetorquers attitude control for differential aerodynamic force application to nanosatellite formation flying construction and maintenance. Advances in the Astronautical Sciences 2020, 170, 385-397.

4. Kumar, B.; Ng, A.; Yoshihara, K.; De Ruiter, A. Differential drag as a means of spacecraft formation control. IEEE Transactions on Aerospace and Electronic Systems 2011, 47, 1125-1135. doi:10.1109/TAES.2011.5751247. 
5. Pérez, D.; Bevilacqua, R. Lyapunov-based Spacecraft Rendezvous Maneuvers using Differential Drag. Proc. AIAA Guidance, Navigation, and Control Conference, Portland, Oregon, 2011, pp. AIAA 2011-6630.

6. Varma, S.; Kumar, K. Multiple satellite formation flying using differential aerodynamic drag. J. Spacecraft and Rockets 2012, 49, 325-336. doi:10.2514/1.52395.

7. Horsley, M.; Nikolaev, S.; Pertica, A. Small satellite rendezvous using differential lift and drag. J. Guidance Control Dyn. 2013, 36, 445-453. doi:10.2514/1.57327.

8. Kumar, K.; Misra, A.; Varma, S.; Reid, T.; Bellefeuille, F. Maintenance of satellite formations using environmental forces. Acta Astronautica 2014, 102, 341-354. doi:10.1016/j.actaastro.2014.05.001.

9. Dellelce, L.; Kerschen, G. Optimal propellantless rendez-vous using differential drag. Acta Astronaut. 2015, 109, 112-123.

10. Ivanov, D.; Monakhova, U.; Ovchinnikov, M. Nanosatellites swarm deployment using decentralized differential drag-based control with communicational constraints. Acta Astronautica 2019, 159, 646-657. doi:10.1016/j.actaastro.2019.02.006.

11. Ivanov, D.; Biktimirov, S.; Chernov, K.; Kharlan, A.; Monakhova, U.; Pritykin, D. Writing with Sunlight: Cubesat formation control using aerodynamic forces. Proc. Int. Astronautical Congress, IAC, 2019, Vol.2019-October.

12. Tang, A.; Wu, X. LEO satellite formation flying via differential atmospheric drag. Int. Journal of Space Science and Engineering 2019, 5, 289-320. doi:10.1504/IJSPACESE.2019.105054.

13. Shouman, M.; Bando, M.; Hokamoto, S. Output regulation control for satellite formation flying using differential drag. J. Guidance Control Dyn. 2019, 42, 2220-2232. doi:10.2514/1.G004219.

14. Smith, B.; Capon, C.; Brown, M. Ionospheric drag for satellite formation control. J. Guidance Control Dyn. 2019 42, 2590-2599. doi:10.2514/1.G004404.

15. Traub, C.; Herdrich, G.; Fasoulas, S. Influence of energy accommodation on a robust spacecraft rendezvous maneuver using differential aerodynamic forces. CEAS Space Journal 2020, 12, 43-63. doi:10.1007/s12567-019-00258-8.

16. Traub, C.; Romano, F.; Binder, T.; Boxberger, A.; Herdrich, G.; Fasoulas, S.; Roberts, P.; Smith, K.; Edmondson, S.; Haigh, S.; Crisp, N.; Oiko, V.; Lyons, R.; Worrall, S.; Livadiotti, S.; Becedas, J.; Gonzlez, G.; Dominguez, R.; Gonzlez, D.; Ghizoni, L.; Jungnell, V.; Bay, K.; Morsbl, J.; Garcia-Almiana, D.; Rodriguez-Donaire, S.; Sureda, M.; Kataria, D.; Outlaw, R.; Villain, R.; Perez, J.; Conte, A.; Belkouchi, B.; Schwalber, A.; Heierer, B. On the exploitation of differential aerodynamic lift and drag as a means to control satellite formation flight. CEAS Space Journal 2020, 12, 15-32. doi:10.1007/s12567-019-00254-y.

17. Leonard, C. Formationkeeping of Spacecraft via Differential Drag. Master's thesis, Massachusetts Inst. Technol., Cambridge, MA, USA, 1986.

18. Hill, G.W. Researches in the Lunar Theory. American J. Mathematics 1878, 1, 5-26. doi:10.2307/2369430.

19. Clohessy, W.; Wiltshire, R. Terminal guidance system for satellite rendezvous. J. Aerospace Sciences 1960, pp. 653-658. doi:10.2514/8.8704.

20. Sedwick, R.; Miller, D.; Kong, E. Mitigation of Differential Perturbations. J. Astronautical Sciences 1999, 47, 309-331.

21. Schweighart, S.; Sedwick, R. High-Fidelity Linearized J2 Model for Satellite Formation Flight. J. Guid. Control. Dyn. 2002, 25, 1073-1080.

22. Schlanbusch, R.; Kristiansen, R.; Nicklasson, P. Spacecraft formation reconfiguration with collision avoidance. Automatica 2011, 47, 1443-1449. doi:10.1016/j.automatica.2011.02.014.

23. Monakhova, U.; Ivanov, D. Formation of a swarm of nanosatellites using decentralized aerodynamic control, taking into account communication constraints. M.V. Keldysh Institute preprints 2018. (in Russian), doi:10.20948/prepr-2018-151.

24. Kwakernaak, H.; Sivan, R. Linear Optimal Control Systems; Wiley-Interscience, 1972.

25. Schweighart, S.; Sedwick, R. Cross-Track Motion of Satellite Formations in the Presence of J2 Disturbances. J. Guid. Control. Dyn. 2005, 28, 824-826. doi:10.2514/1.12387.

26. Wang, D.; Wu, B.; Poh, E.K. Satellite Formation Flying Relative Dynamics, Formation Design, Fuel Optimal Maneuvers and Formation Maintenance, S.G. Tzafestas ed.; Vol. 87, Intelligent Systems, Control and Automation: Science and Engineering, Springer, 2017. doi:10.1007/978-981-10-2383-5.

27. Bevilacqua, R.; Hall, J., S.; Romano, M. Multiple Spacecraft Assembly Maneuvers by Differential Drag and Low Thrust Engines. Celestial Mechanics and Dynamical Astronomy 2010, 106, 69-88. doi:10.1007/s10569-009-9240-3.

28. Bevilacqua, R.; Romano, M. Rendezvous Maneuvers of Multiple Spacecraft by Differential Drag under J2 Perturbation J. Guidance, Control and Dynamics 2008, 31, 1595-1607. doi:10.2514/1.36362. 
29. Kim, D.Y.; Woo, B.; Park, S.Y.; Choi, K.H. Hybrid optimization for multiple-impulse reconfiguration trajectories of satellite formation flying. Advances in Space Research 2009, 44, 1257-1269. doi:10.1016/j.asr.2009.07.029.

30. Vaddi, S.; Alfriend, K.; Vadali, S.; Sengupta, P. Formation establishment and reconfiguration using impulsive control. J. Guidance Control Dyn. 2005, 28, 262-268. doi:10.2514/1.6687.

31. Vaddi, S. Modeling and Control of Satellite Formations. PhD thesis, Department of Aerospace Engineering, Texas A\&M University, 2003.

32. Omar, S.; Bevilacqua, R. Guidance, Navigation, and Control Solutions for Spacecraft Re-Entry Point Targeting Using Aerodynamic Drag. Acta Astronautica 2019, 155, 389-405. doi:10.1016/j.actaastro.2018.10.016.

33. Nair, G.; Evans, R. Exponential stabilisability of finite-dimensional linear systems with limited data rates. Automatica 2003, 39, 585-593.

34. Bazzi, L.; Mitter, S. Endcoding complexity versus minimum distance. IEEE Trans. Inform. Theory 2005, 51, 2103-2112.

35. Nair, G.; Fagnani, F.; Zampieri, S.; Evans, R. Feedback control under data rate constraints: an overview. Proc. IEEE 2007, 95, 108-137.

36. Matveev, A.; Savkin, A. Estimation and Control over Communication Networks; Birkhäuser: Boston, 2009.

37. Andrievsky, B.; Matveev, A.; Fradkov, A. Control and estimation under information constraints: Toward a unified theory of control, computation and communications. Automation and Remote Control 2010, 71, 572-633. (Translated from Avtomatika i Telemekhanika, No. 4, 2010, P. 34-99).

38. Renga, A.; Grassi, M.; Tancredi, U. Relative navigation in LEO by carrier-phase differential GPS with intersatellite ranging augmentation. Int. J. Aerosp. Eng. 2013, 2013.

39. Fradkov, A.L.; Miroshnik, I.V.; Nikiforov, V.O. Nonlinear and Adaptive Control of Complex Systems; Kluwer: Dordrecht, 1999.

40. Andrievsky, B.; Kudryashova, E.; Kuznetsov, N.; Kuznetsova, O. Aircraft wing rock oscillations suppression by simple adaptive control. Aerosp. Sci. Technol. 2020, 105, 1-10. doi:10.1016/j.ast.2020.106049.

41. Hespanha, J.P.; Naghshtabrizi, P.; Xu, Y.A Survey of Recent Results in Networked Control Systems. Proc. IEEE 2007, 95, 138-162.

42. Andrievsky, B.; Fradkov, A.L. Control and observation via communication channels with limited bandwidth. Gyroscopy and Navigation 2010, 1, 126-133. (Translated from Giroskopiya i Navigatsiya, No. 4, 2009, pp. $103-$ 114).

43. Nair, G.; Evans, R. Stabilizability of stochastic linear systems with finite feedback data rates. SIAM J. Control Optim 2004, 43, 413-436.

44. Nair, G.; Evans, R.; Mareels, I.; Moran, W. Topological feedback entropy and nonlinear stabilization. IEEE Trans. Automat. Contr. 2004, 49, 1585-1597.

45. Fradkov, A.; Andrievsky, B.; Evans, R. Chaotic observer-based synchronization under information constraints. Physical Review E 2006, 73, 066209.

46. Cover, T.; Thomas, J. Elements of Information Theory; John Wiley \& Sons, Inc.: New York, Chichester, Brisbane, Toronto, Singapore, 1991.

47. Rizzo, L. Effective Erasure Codes for Reliable Computer Communication Protocols. Computer Communication Review 1997, 27, 24-36. doi:10.1145/263876.263881.

48. Tatikonda, S.; Mitter, S. Control Over Noisy Channels. IEEE Trans. Automat. Contr. 2004, 49, 1196-1201.

49. Shokrollahi, A. Raptor codes. IEEE Trans. Inform. Theory 2006, 52, 2551-2567.

50. Köetter, R.; Kschischang, F. Coding for Errors and Erasures in Random Network Coding. IEEE Trans. Inform. Theory 2008, 54, 3579-3591. doi:10.1109/TIT.2008.926449.

51. Patterson, S.; Bamieh, B.; El Abbadi, A. Convergence Rates of Distributed Average Consensus With Stochastic Link Failures. IEEE Trans. Automat. Contr. 2010, 55, 880-892. doi:10.1109/TAC.2010.2041998.

52. Diwadkar, A.; Vaidya, U. Robust synchronization in nonlinear network with link failure uncertainty. Proc. 50th IEEE Conf. Decision and Control and European Control Conference (CDC-ECC 2011); IEEE: Orlando, FL, USA, 2011; pp. 6325-6330.

53. Wang, J.; Yan, Z. Coding scheme based on spherical polar coordinate for control over packet erasure channel. Int. J. Robust and Nonlinear Control 2014, 24, 1159-1176.

54. Zhang, H.; Lee, S.; Li, X.; He, J. EEG self-adjusting data analysis based on optimized sampling for robot control. Electronics (Switzerland) 2020, 9, 1-17. doi:10.3390/electronics9060925. 
55. Jeon, S.; Park, C.; Seo, D. The multi-station based variable speed limit model for realization on urban highway. Electronics (Switzerland) 2020, 9. doi:10.3390/electronics9050801.

56. Tsypkin, Y. Stability and sensitivity of nonlinear sampled data systems. In Sensitivity Methods in Control Theory; Radanovic, L., Ed.; Pergamon, 1966; pp. 46 - 66. doi:https://doi.org/10.1016/B978-1-4831-9822-4.50007-8.

57. Pogromsky, A.; Matveev, A. A non-quadratic criterion for stability of forced oscillation. Systems \& Control Letters 2013, 62, 408-412.

58. Dudkowski, D.; Jafari, S.; Kapitaniak, T.; Kuznetsov, N.V.; Leonov, G.A.; Prasad, A. Hidden attractors in dynamical systems. Physics Reports 2016, 637, 1 - 50. doi:http://dx.doi.org/10.1016/j.physrep.2016.05.002.

59. Kuznetsov, N. Theory of hidden oscillations and stability of control systems. J. Computer and Systems Sciences International 2020, 59, 647-668. doi:10.1134/S1064230720050093.

60. Xia, Y.Q.; Gao, Y.L.; L.-P., Y.; Fu, M.Y. Recent progress in networked control systems - A survey. Intern. J. Automation and Computing 2015, 12, 343-367. doi:10.1007/s11633-015-0894-x.

61. Nair, G.; Evans, R. State estimation via a capacity-limited communication channel. Proc. 36th IEEE Conference on Decision and Control (CC'97); , 1997; pp. 866-871.

62. De Persis, C. $n$-Bit stabilization of $n$-dimensional nonlinear systems in feedforward form. IEEE Trans. Automat. Contr. 2005, 50,299-311.

63. Liberzon, D.; Hespanha, J. Stabilization of nonlinear systems with limited information feedback. IEEE Trans. Automat. Contr. 2005, 50,910-915.

64. Matveev, A.; Proskurnikov, A.; Pogromsky, A.; Fridman, E. Comprehending complexity: Data-rate constraints in large-scale networks. IEEE Trans. Automat. Contr. 2019, 64, 4252-4259. cited By 0, doi:10.1109/TAC.2019.2894369.

65. Voortman, Q.; Pogromsky, A.; Matveev, A.; Nijmeijer, H. Data-rate constrained observers of nonlinear systems. Entropy 2019, 21. doi:10.3390/e21030282.

66. Matveev, A.; Pogromsky, A. Observation of nonlinear systems via finite capacity channels, Part II: Restoration entropy and its estimates. Automatica 2019, 103, 189-199. cited By 2, doi:10.1016/j.automatica.2019.01.019.

67. Åström, K.J.; Bernhardsson, B.M. Comparison of Riemann and Lebesgue sampling for first order stochastic systems. Proc. 41st IEEE Conf. on Decision \& Control (CDC 2002); , 2002; pp. 2011-2016.

68. Yu, H.; Antsaklis, P.J. Output Synchronization of Networked Passive Systems With Event-Driven Communication. IEEE Trans. Automat. Contr. 2014, 59, 750-756.

69. Margun, A.; Furtat, I.; Zimenko, K.; Kremlev, A. Event-triggered output robust controller. Proc. 25th Mediterranean Conf. Control Automation (MED 2017), 2017, pp. 625-630. doi:10.1109/MED.2017.7984187.

70. Li, K.; Baillieu, J. Robust quantization for digital finite communication bandwidth (DFCB) control. IEEE Trans. Automat. Contr. 2004, 49, 1573-1584.

71. Gabor, G.; Gyorfi, Z. Recursive Source Coding; Springer-Verlag: NY,1986.

72. Tatikonda, S.; Sahai, A.; Mitter, S. Control of LQG Systems Under Communication Constraints. Proc. 37th IEEE Conf. Decision and Control; IEEE: Tampa, Florida USA, 1998; Vol. WP04, pp. 1165-1170.

73. Brockett, R.; Liberzon, D. Quantized feedback stabilization of linear systems. IEEE Trans. Automat. Contr. 2000, 45, 1279-1289.

74. Liberzon, D. Hybrid feedback stabilization of systems with quantized signals. Automatica 2003, 39, 1543-1554.

75. Tatikonda, S.; Mitter, S. Control under communication constraints. IEEE Trans. Automat. Contr. 2004, 49, 1056-1068.

76. Fradkov, A.; Andrievsky, B.; Ananyevskiy, M. State estimation and synchronization of pendula systems over digital communication channels. Europ. Phys. J.: Special Topics 2014, 223, 773-793. doi:10.1140/epjst/e2014-02140-0.

77. Fradkov, A.L.; Andrievsky, B.; Evans, R.J. Adaptive Observer-Based Synchronization of Chaotic Systems with First-Order Coder in Presence of Information Constraints. IEEE Trans. Circuits Syst. I 2008, 55, 1685-1694.

78. Fradkov, A.; Andrievsky, B.; Evans, R. Synchronization of passifiable Lurie systems via limited-capacity communication channel. IEEE Trans. Circuits Syst. I 2009, 56, 430-439.

79. Moreno-Alvarado, R.; Rivera-Jaramillo, E.; Nakano, M.; Perez-Meana, H. Simultaneous Audio Encryption and Compression Using Compressive Sensing Techniques. Electronics 2020, 9, 863. doi:10.3390/electronics9050863.

80. Goodman, D.; Gersho, A. Theory of an adaptive quantizer. IEEE Trans. Commun. 1974, COM-22, 1037-1045.

81. Andrievsky, B.; Fradkov, A. Adaptive coding for position estimation in formation flight control. Proc. IFAC Workshop Adaptation and Learning in Control and Signal Processing (ALCOSP 2010); , 2010; pp. $72-76$. 
82. Fradkov, A.; Andrievsky, B.; Peaucelle, D. Estimation and control under information constraints for LAAS helicopter benchmark. IEEE Trans. Contr. Syst. Technol. 2010, 18, 1180-1187.

83. Gomez-Estern, F.; Canudas de Wit, C.; Rubio, F. Adaptive delta modulation in networked controlled systems with bounded disturbances. IEEE Trans. Automat. Contr. 2011, 56, 129-134.

84. La Scala, B.; Evans, R. Minimum necessary data rates for accurate track fusion. Proc. 44th IEEE Conference on Decision and Control, and the European Control Conference; IEEE: Seville, Spain, 2005; pp. 6966-6971.

85. Evans, R.; Krishnamurthy, V.; Nair, G.; Sciacca, L. Networked sensor management and data rate control for tracking maneuvering targets. IEEE Trans. Signal Processing 2005, 53, 1979-1991.

86. Tomashevich, S.; Andrievsky, B.; Fradkov, A.L. Formation control of a group of unmanned aerial vehicles with data exchange over a packet erasure channel. Proc. 1st IEEE Intern. Conf. Industrial Cyber-Physical Systems (ICPS 2018). IEEE, 2018, pp. 38-43. doi:10.1109/ICPHYS.2018.8387634.

87. Andrievsky, B. Numerical evaluation of controlled synchronization for chaotic Chua systems over the limited-band data erasure channel. Cybernetics and Physics 2016, 5, 3-51. 